\title{
METRIC AND ALGEBRAIC PERTURBATIONS OF FUNCTION ALGEBRAS
}

\author{
by KRZYSZTOF JAROSZ
}

(Received 27th September 1982)

Let $A$ and $B$ be function algebras. We generalise the Nagasawa theorem by proving that the Banach-Mazur distance between the underlying Banach spaces of $A$ and $B$, is close to one if and only if they are almost isomorphic, that is if and only if there is a linear map $T$ from $A$ onto $B$ such that $\left\|T^{-1}(T f \cdot T g)-f g\right\| \leqq \varepsilon\|f\|\|g\|$.

In this paper we define two kinds of perturbations of the algebraic structure of a Banach algebra and prove that they coincide for function algebras.

By an $\varepsilon$-perturbation (algebraic perturbation) of a Banach algebra $(A, \cdot)$ we mean an associative multiplication $\times$ defined on the Banach space $A$ such that

$$
\|f \times g-f \cdot g\| \leqq \varepsilon\|f\|\|g\| \quad \text { for all } f, g \text { in } A \text {. }
$$

The $\varepsilon$-perturbations were studied by R. V. Kadison, D. Kastler [4] and J. Phillips [6], and in a more general situation by B. E. Johnson [3] and I. Raeburn and J. L. Taylor [7]. They investigate whether all multiplications on a Banach algebra $(A, \cdot)$ near the given multiplication share any of the properties of the original one. The best possible situation happens if every sufficiently small perturbation produces a new algebra which is algebraically isomorphic to the original one. Such an algebra is called rigid or algebraically stable. Johnson, and Raeburn and Taylor proved that an algebra $A$ is rigid if the second and the third groups of cohomology of $A$ vanish. Johnson also gave several examples and counter-examples of rigid Banach algebras, for example he obtained rigidness of the algebra $C(S)$ of all continuous functions on a compact metric space $S$. Using another method $R$. Rochberg [8] proved this and some other results concerning $\varepsilon$-perturbations. Rochberg investigated perturbations of function algebras, that is, of the commutative Banach algebras with unit such that $\left\|f^{2}\right\|=\|f\|^{2}$ for any element $f$ of the algebra. Some of his results were generalised in [2].

For function algebras one can define, in a natural way, another type of perturbation. To this end, let us recall the Nagasawa theorem.

Theorem ([5]). Two function algebras $A$ and $B$ are isometric if and only if they are algebraically isomorphic.

Accordingly, we shall call a function algebra $B$ an $\varepsilon$-metric perturbation of a function algebra $A$ if and only if the Banach-Mazur distance between the underlying Banach 
spaces of $A$ and $B$ is smaller than $1+\varepsilon$. A function algebra $A$ will be called metrically stable if and only if all sufficiently small metric perturbations of $A$ are isomorphic with $A$. Let us now fix some terminology. If $A$ and $B$ are Banach algebras and if $T$ is a homomorphism of the underlying Banach spaces of $A$ into the underlying Banach spaces of $B$ then we call $T$ a linear homomorphism; if $T$ is also a homomorphism of algebras then we call it an algebraic homomorphism.

Notice that if $T$ is any linear isomorphism between the underlying Banach spaces of algebras $A$ and $B$ then $T$ defines another multiplication $\times$ on $A$ :

$$
f \times g=T^{-1}(T f \cdot T g) \text { for } f, g \text { in } A .
$$

Notice also that if $\times$ is a commutative $\varepsilon$-perturbation of the Banach algebra $(A, \times)$ then the Gelfand transformation of the algebra $(A, \mathrm{x})$ is a linear map from a Banach space $A$ into a function algebra $C\left(M\left(A_{\times}\right)\right), M\left(A_{\times}\right)$being the maximal ideal space of the algebra $(A, \times)$.

Our main theorem is

Theorem 1. For any function algebra $(A, \cdot)$ the following holds:

(i) if $\times$ is an $\varepsilon$-algebraic perturbation of $A$ and if $\varepsilon<1$, then the multiplication $\times$ is commutative, the Gelfand transformation of the algebra $(A, \times)$ is an isomorphism from $A$ onto a closed subalgebra $B$ of $C\left(M\left(A_{\times}\right)\right)$and the Banach-Mazur distance between $A$ and $B$ is less than $(1+\varepsilon) /(1-\varepsilon)$;

(ii) if a continuous linear isomorphism $T$ between $A$ and a function algebra $B$ defines an $\varepsilon$-metric perturbation of $A$, then there exists a linear isomorphism $\widetilde{T}$ between $A$ and $B$ such that $\|\widetilde{T}\|\left\|\tilde{T}^{-1}\right\| \leqq 1+\varepsilon^{\prime}(\varepsilon)$ and such that the multiplication on $A$ defined by $\tilde{T}$ is an $\varepsilon^{\prime}(\varepsilon)-$ algebraic perturbation of the original multiplication of $A$, and $\varepsilon^{\prime}(\varepsilon) \rightarrow 0$ as $\varepsilon \rightarrow 0$. Moreover, if $T e_{A}=e_{B}$ then $\tilde{T}$ equals $T$.

This theorem was proved by R. Rochberg ([8], p. 102) in the special case when the Shilov and the Choquet boundaries of $A$ coincide and when every point of the Shilov boundary of $A$ is a $G_{\delta}$ set. Before proving this theorem let us formulate, as immediate consequences, the following theorems, the first of which generalises the Nagasawa theorem.

Theorem 2. Function algebras $A$ and $B$ are $\varepsilon$-almost isometric (this means that there exists $T: A \rightarrow B:\|T\|\left\|T^{-1}\right\| \leqq 1+\varepsilon$ ) if and only if they are $\varepsilon^{\prime}$-almost algebraically isomorphic (this means that there exists a linear isomorphism $T$ of $A$ onto $B$ such that $\| T^{-1}(T f \cdot T g$ ) $-f g\left\|\leqq \varepsilon^{\prime}\right\| f\|\| g \|$ for all $f, g$ in $A$ ), where $\varepsilon$ and $\varepsilon^{\prime}$ tend to zero simultaneously.

Proof. The "only if" part is just Theorem 1 (ii). To get the converse implication assume

$$
\|f \times g-f \cdot g\| \leqq \varepsilon\|f\|\|g\| \text { for all } f, g \text { in } A \text {, }
$$

where

$$
f \times g=T^{-1}(T f \cdot T g)
$$


By Theorem 1. (i) there is a continuous algebra isomorphism ^ from $(A, \mathrm{x})$ onto some function algebra $C$. The maps ${ }^{\wedge} \circ T^{-1}$ and $T \circ^{\wedge-1}$ being algebraic isomorphisms between two function algebras $B$ and $C$ are isometric and hence

$$
\|T\|\left\|T^{-1}\right\|=\left\|^{-}\right\|\left\|^{-1}\right\| \leqq(1+\varepsilon) /(1-\varepsilon) .
$$

Notice that we do not assume the continuity of $T$ but we get it from the condition

$$
\left\|T^{-1}(T f \cdot T g)-f \cdot g\right\| \leqq \varepsilon\|f\|\|g\| \quad \text { for } \quad \varepsilon<1
$$

Theorem 3. A function algebra $A$ is algebraically stable if and only if it is metrically stable.

Before passing to the proof of Theorem 1 we derive one more theorem which we shall need later. This theorem is valid for arbitrary Banach algebras not necessarily function algebras and seems to be interesting in itself. It allows for a significant simplification when checking whether a new multiplication is an \&-perturbation of the original multiplication of the Banach algebra.

Theorem 4. Let $A$ be a Banach space and let,$\times$ be two multiplications on $A$ with identity elements $e$ and $\tilde{e}$. Assume that $(A, \cdot)$ is a Banach algebra. There is a function $c: \mathbb{R}^{+} \rightarrow \mathbb{R}^{+}$such that $\lim c(\varepsilon)=0$ as $\varepsilon \rightarrow 0$ and if

then

$$
|\|f \times g\|-\|f \cdot g\|| \leqq \varepsilon\|f\|\|g\| \text { for all } f, g \text { in } A,
$$

$$
\left\|e^{-1} \times f \times g-f \cdot g\right\| \leqq c(\varepsilon)\|f\|\|g\| \text { for any } f, g \text { in } A \text {; }
$$

where $e^{-1}$ is the inverse element of $e$ in the algebra $(A, \mathrm{x})$.

Proof. Replace $f$ in (2) by the element $\exp (\lambda f)$ where $\lambda$ is a complex number and exp is the exponential function in the Banach algebra $(A, \cdot)$, and replace $g$ by $g \cdot \exp (-\lambda f)$. We get

$$
\begin{aligned}
\mid\|g\|-\|\exp \lambda f \times[\exp (-\lambda f) \cdot g]\| & \leqq \varepsilon\|g\|\|\exp (-\lambda f)\| \cdot\|\exp \lambda f\| \\
& \leqq \varepsilon\|g\| \exp (2|\lambda|\|f\|)
\end{aligned}
$$

This gives

$$
\begin{aligned}
\|g\| & {[1+\varepsilon \exp (2|\lambda|\|f\|)] } \\
\quad & \geqq\|\exp (\lambda f) \times[\exp (-\lambda f) \cdot g]\| \\
& =\left\|\left(e+\lambda f / 1 !+\lambda^{2} f^{2} / 2 !+\ldots\right) \times\left(g-\lambda f g / 1 !+\lambda^{2} f^{2} g / 2 !-\ldots\right)\right\| \\
& =\left\|e \times g-(f \times g-e \times(f g)) \lambda / 1 !+(\ldots) \lambda^{2} / 2 !+\ldots\right\| .
\end{aligned}
$$

EMS-E 
Now let $F$ be any linear functional on the Banach space $A$ of norm equal to one. We have

$$
\begin{aligned}
& \left|F(e \times g)-\lambda / 1 ! F(f \times g-e \times(f g))+\lambda^{2} / 2 ! F(\ldots)+\ldots\right| \\
& \quad \leqq\|g\|[\varepsilon \exp (2|\lambda|\|f\|)+1] .
\end{aligned}
$$

This shows that the modulus of the entire function

$$
\varphi(\lambda)=F(e \times g)-\lambda / 1 ! F(f \times g-e \times(f g))+\lambda^{2} / 2 ! F(\ldots)-\ldots
$$

on the unit disc $D=\{\lambda \in \mathbb{C}:|\lambda|<1\}$ is not greater than $\|g\|[\varepsilon \exp 2\|f\|+1]$, hence the first derivative at the point zero of this function has modulus not greater than this constant too. Because $F$ is an arbitrary functional of norm 1, it follows that

$$
\|f \times g-e \times(f \cdot g)\| \leqq\|g\|[1+\varepsilon \exp 2\|f\|] \quad \text { for any } f, g \text { in } A .
$$

Fix elements $f$ and $g$ in $A$ both of norm 1, and let $\alpha=-\frac{1}{2} \log \varepsilon>0$. From (4) we get

$$
\begin{aligned}
\|f \times g-e \times(f \cdot g)\| & =\|(\alpha f) \times(g / \alpha)-e \times((\alpha f) \cdot(g / \alpha))\| \\
& \leqq \alpha^{-1}[\varepsilon \exp 2 \alpha+1]=-4 / \log \varepsilon
\end{aligned}
$$

for $0<\varepsilon<1$. This shows the existence of a positive number $\varepsilon_{1}=\varepsilon_{1}(\varepsilon)=-4 / \log \varepsilon$ such that $\varepsilon_{1}(\varepsilon) \rightarrow 0$ as $\varepsilon \rightarrow 0$ and

$$
\|f \times g-e \times(f \cdot g)\| \leqq \varepsilon_{1}\|f\|\|g\| \text { for any } f, g \text { in } A .
$$

Now let us estimate the norm of the unit $\tilde{e}$ of the algebra $(A, \times)$. From (2) we have

$$
\text { i }\|e\|-\|\tilde{e}\||=|\|e \times \tilde{e}\|-\|e \cdot \tilde{e}\| \mid \leqq \varepsilon\|\tilde{e}\|,
$$

and hence

$$
\|\tilde{e}\| \leqq 1 /(1-\varepsilon) .
$$

Setting $f=g=\tilde{e}$ in (5) we find

$$
\|\tilde{e}-e \times(\tilde{e} \cdot \tilde{e})\| \leqq \varepsilon_{1}\|\tilde{e}\|^{2} \leqq \varepsilon_{1} /(1-\varepsilon)^{2} .
$$

By (2)

$$
\|f \times g\| \leqq(1+\varepsilon)\|f\|\|g\| \text { for all } f, g \text { in } A
$$

which implies that if $(1+\varepsilon) \varepsilon_{1}\|\tilde{e}\|^{2}<1$ then $e$ is an invertible element of $(A, \times)$. Further if

$$
\|\tilde{e}-x\| \leqq \varepsilon_{1}\|\tilde{e}\|^{2}<1 /(1+\varepsilon)
$$


then

$$
\begin{aligned}
\left\|x^{-1}\right\| & \leqq\|\tilde{e}\|+\sum_{n=1}^{\infty} \|(\underbrace{(\tilde{e}-x) \times \ldots \times(\tilde{e}-x)}_{n \text { times }} \| \\
& \leqq\|\tilde{e}\|+\sum_{n=1}^{\infty}(1+\varepsilon)^{n-1}\left(\varepsilon_{1}\|\tilde{e}\|^{2}\right)^{n} \\
& \leqq(1-\varepsilon)^{-1}\left(1-\varepsilon_{1}(1-\varepsilon)\|\tilde{e}\|^{2}\right)^{-1}
\end{aligned}
$$

so that

$$
\left\|e^{-1}\right\|=\left\|(e \times(\tilde{e} \cdot \tilde{e}))^{-1} \times(\tilde{e} \cdot \tilde{e})\right\| \leqq(1+\varepsilon)(1-\varepsilon)^{-3}\left(1-\varepsilon_{1}(1+\varepsilon)(1-\varepsilon)^{-2}\right)^{-1}
$$

provided $\varepsilon_{1}(1+\varepsilon)(1-\varepsilon)^{-2}<1$. Moreover, for each $f$ and $g$ in $A$ we have

$$
\begin{aligned}
& \left\|e^{-1} \times f \times g-f \cdot g\right\| \\
& \quad \leqq(1+\varepsilon)\left\|e^{-1}\right\|\|f \times g-e \times(f \cdot g)\| \\
& \quad \leqq(1+\varepsilon)^{2}(1-\varepsilon)^{-3}\left(1-\varepsilon_{1}(1+\varepsilon)(1-\varepsilon)^{-2}\right)^{-1} \varepsilon_{1}\|f\|\|g\| \\
& \quad \leqq-\frac{4(1+\varepsilon)^{2}(1-\varepsilon)^{-3}}{\log \varepsilon+4(1+\varepsilon)(1-\varepsilon)^{-2}}\|f\|\|g\|=c(\varepsilon)\|f\|\|g\| .
\end{aligned}
$$

Corollary. Let $(A, \cdot)$ be a Banach algebra with identity. Assume that on the underlying Banach space of $A$ there is defined another multiplication $\times$ such that $\|f \times g\|=\|f \cdot g\|$ for all $f, g$ in $A$. If both multiplications $\times$ and possess the same identity, then they coincide.

\section{Proof of Theorem 1.}

Let us first prove the easy part of Theorem 1 . Let $\times$ be an $\varepsilon$-perturbation of $A$. For any $f$ in $A$ we have

$$
\|f \times f-f \cdot f\| \leqq \varepsilon\|f\|^{2}
$$

and hence

$$
(1-\varepsilon)\|f\|^{2} \leqq\|f \times f\|=(1+\varepsilon)\|f\|^{2}
$$

By induction

$$
(1-\varepsilon)^{2^{n}-1}\|f\|^{2^{n}} \leqq\|\underbrace{f \times f \times \ldots \times f \|}_{2^{n} \text { times }}\|(1+\varepsilon)^{2^{n}-1}\|f\|^{2^{n}}
$$

The above inequalities prove 
Proposition 1. If $x$ is an E-perturbation of a function algebra $A$ then the spectral radius of any element $f$ of the algebra $(A, \times)$ is contained between $(1-\varepsilon)\|f\|$ and $(1+\varepsilon)\|f\|$.

From this proposition and the theorem of Hirschfeld-Zelazko [1] we deduce the commutativity of $x$ if $\varepsilon<1$. Denote by ${ }^{\wedge}$ the Gelfand transformation of the algebra $(A, \times)$. By Proposition 1 it is an isomorphism of the algebra $(A, \times)$ onto a closed subalgebra $B$ of $C\left(M\left(A_{\times}\right)\right)$such that

$$
\|\|^{\wedge}-1 \| \leqq(1+\varepsilon) /(1-\varepsilon) .
$$

For the proof of the second part of Theorem 1 we need the following lemma.

Lemma. Let $T$ be a continuous linear isomorphism of a function algebra $A$ onto a function algebra $B$ with $\|T\|=1$ and $\left\|T^{-1}\right\| \leqq 1+\varepsilon<1.5$. Then there exists a dense subset $\Omega$ of the Shilov boundary of $A$ such that for each $x$ in $\Omega$ there is $y$ in the Shilov boundary of $B$ such that

$$
|T f(y)| \geqq|f(x)|-2 \varepsilon\|f\| \text { for all } f \text { in } A \text {. }
$$

Proof. Denote by $\partial A, \partial B$ the Shilov boundaries of $A$ and $B$ and by $\mathrm{Ch} A, \mathrm{Ch} B$ the Choquet boundaries of these algebras. We will call a net $\left(g_{\alpha}\right)$ of the elements of the function algebra $B$ a peaking net at a point $y \in \operatorname{Ch} B$ if and only if

$$
\left\|g_{\alpha}\right\|=g_{\alpha}(y)=1 \text { for all } \alpha
$$

and

$\left(g_{\alpha}\right)$ tends uniformly to zero outside any neighbourhood of $y$.

We shall denote by $\Omega_{y}$ the subset of $\partial A$ consisting of all points $x_{0}$ admitting a net $\left(g_{\alpha}\right) \subset B$ peaking at $y$ and a net $\left(x_{\alpha}\right) \subset \partial A$ converging to $x_{0}$ and such that

$$
\left|T^{-1} g_{\alpha}\left(x_{\alpha}\right)\right| \geqq 1-\varepsilon \quad \text { for all } \alpha .
$$

Since $\|T\|=1$ and because the set $\partial A$ is compact it follows that the set $\Omega_{y}$ is non-void.

Notice now that for any $f$ in $A$ and for a suitable net $\left(\eta_{\alpha}\right)$ of complex numbers of modulus one we have

$$
\lim \sup _{\alpha}\left\|f+\eta_{\alpha} T^{-1} g_{\alpha}\right\| \geqq 1-\varepsilon+\left|f\left(x_{0}\right)\right|
$$

hence

$$
\lim \sup _{\alpha}\left\|T f+\eta_{\alpha} g_{\alpha}\right\| \geqq\left(1-\varepsilon+\left|f\left(x_{0}\right)\right|\right) /(1+\varepsilon) .
$$


Thus, by definition of $\left(g_{\alpha}\right)$ we obtain

$$
|T f(y)| \geqq\left(1-\varepsilon+\left|f\left(x_{0}\right)\right|\right) /(1+\varepsilon)-1 \geqq\left|f\left(x_{0}\right)\right|-2 \varepsilon
$$

for any function $f$ in $A$ of norm 1 .

So, we have proved that (6) is satisfied for any $y$ in $\operatorname{Ch} B$ and any $x_{0}$ in $\Omega_{y}$. It remains to prove that $\Omega_{0}=\bigcup\left\{\Omega_{y}: y \in \mathrm{Ch} B\right\}$ is a dense subset of $\partial A$. Suppose that it is not. Then there exists an open subset $V$ of $\partial A$ such that $\bar{V} \cap \Omega_{0}=\emptyset$. Fix $0<\delta<1-2 \varepsilon$ and an element $f_{1}$ from $A$ such that

$$
\left\|f_{1}\right\|=1 \text { and }\left|f_{1}(x)\right|<\delta \text { for } \quad x \in \partial A-V .
$$

Take $y_{1} \in \mathrm{Ch} B$ such that

$$
\left|T f_{1}\left(y_{1}\right)\right| \geqq 1 /(1+\varepsilon)>1-\varepsilon,
$$

and let $\left(g_{\alpha}\right) \subset B$ be a net peaking at $y_{1}$. For a suitable net $\left(\xi_{\alpha}\right)$ of complex numbers of modulus one we have

$$
\limsup _{\alpha}\left\|T f_{1}+\xi_{\alpha} g_{\alpha}\right\|>1-\varepsilon+1
$$

Hence

$$
\lim \sup _{\alpha}\left\|f_{1}+\xi_{\alpha} T^{-1} g_{\alpha}\right\|>2-\varepsilon
$$

so by the definition of $f_{1}$, there exists a net $\left(x_{\alpha}\right) \subset V$ such that

$$
\limsup \left|T^{-1} g_{\alpha}\left(x_{\alpha}\right)\right|>1-\varepsilon
$$

Because $\bar{V}$ is a compact subset of $\partial A$ we can assume that the net $\left(x_{\alpha}\right)$ converges to $x_{0} \in \bar{V}$, which leads in view of (8) to the conclusion that $x_{0} \in \bar{V} \cap \Omega_{0}$. But this contradicts the assumption $\bar{V} \cap \Omega_{0}=\emptyset$ and therefore proves the lemma.

Let us now return to the proof of the second part of Theorem 1 . To this end let $A$ and $B$ be function algebras and let $T$ be a continuous linear isomorphism from $A$ onto $B$ such that $\|T\|\left\|T^{-1}\right\| \leqq 1+\varepsilon$. Without loss of generality, we can assume that $\|T\|=1$ and $\left\|T^{-1}\right\|=1+\varepsilon$. From the lemma, for any $f, g$ in $A$, we have

$$
\begin{aligned}
\|T f \cdot T g\| & =\sup _{y \in \partial B}|T f(y) T g(y)| \geqq \sup _{x \in \Omega}(|f(x)|-2 \varepsilon\|f\|)(|g(x)|-2 \varepsilon\|g\|) \\
& =\|f g\|-4 \varepsilon\|f\|\|g\|+4 \varepsilon^{2}\|f\|\|g\|
\end{aligned}
$$

so that

$$
\|T f \cdot T g\|-\|f g\| \geqq-4 \varepsilon\|f\|\|g\|
$$

for $0 \leqq \varepsilon<\frac{1}{2}$ 
Now put $T_{1}=T^{-1} /\left\|T^{-1}\right\|$ and $f_{1}=T_{1}^{-1} f, g_{1}=T_{1}^{-1} g$. From the lemma, in the same manner as above we derive

$$
\begin{aligned}
\|f g\|-\left\|T_{1}^{-1} f \cdot T_{1}^{-1} g\right\| & =\left\|T_{1} f_{1} \cdot T_{1} g_{1}\right\|-\left\|f_{1} g_{1}\right\|=-4 \varepsilon\left\|f_{1}\right\|\left\|g_{1}\right\| \\
& \geqq-4 \varepsilon(1+\varepsilon)^{2}\|f\|\|g\|,
\end{aligned}
$$

Hence

$$
\begin{aligned}
\|f g\|-\|T f T g\| & \geqq-4 \varepsilon(1+\varepsilon)^{2}\|f\|\|g\|-\left(\|T f T g\|-\left\|T_{1}^{-1} f T_{1}^{-1} g\right\|\right) \\
& \geqq-11 \varepsilon\|f\|\|g\|
\end{aligned}
$$

for $0 \leqq \varepsilon<\frac{1}{2}$.

We get

$$
|\|f \times g\|-\|f \cdot g\||=12 \varepsilon\|f\|\|g\|
$$

where $f \times g=T^{-1}(T f \cdot T g)$.

Now denote by $\tilde{e}, e_{A}, e_{B}$ the units of the algebras $(A, \times),(A, \cdot)$ and $B$, respectively. Notice that $T \tilde{e}=e_{B}$. From (9) and Theorem 4 we have

$$
\left\|e_{A}^{-1} \times f \times g-f \cdot g\right\| \leqq \varepsilon^{\prime}\|f\|\|g\|
$$

where $e_{A}^{-1}$ is the inverse of the element $e_{A}$ in the algebra $(A, \times)$. Let us define an operator $S: A \rightarrow A$ by $S f=e_{A}^{-1} \times f$. From (9) and (10) we see that

$$
\begin{aligned}
|\|S f\|-\|f\|| & =\left|\left\|e_{A}^{-1} \times f\right\|-\|f\|\right| \leqq\left|\left\|e_{A}^{-1} \times f \times \tilde{e}\right\|-\|f \cdot \tilde{e}\|\right|+|\|f \cdot \tilde{e}\|-\|f \times \tilde{e}\|| \\
& =\left(\varepsilon^{\prime}+12 \varepsilon\right)\|\tilde{e}\|\|f\| \leqq\left(\varepsilon^{\prime}+12 \varepsilon\right)(1+\varepsilon)\|f\| .
\end{aligned}
$$

Hence

$$
\|S\|\left\|S^{-1}\right\| \leqq 1+c_{1}(\varepsilon) \quad \text { with } \quad \lim c_{1}(\varepsilon)=0 \quad \text { as } \quad \varepsilon \rightarrow 0 .
$$

Let $\tilde{T}=T \cdot S$. By (11), since $\|T\|\left\|T^{-1}\right\|=1+\varepsilon$, we have $\|\tilde{T}\|\left\|\tilde{T}^{-1}\right\| \leqq 1+c_{2}(\varepsilon)$ with $\lim c_{2}(\varepsilon)=0$ as $\varepsilon \rightarrow 0$. Moreover $\widetilde{T} e_{A}=e_{B}$. Hence by following the same argument that was applied above for $T$ we get

$$
\|f \circ g-f \cdot g\| \leqq c(\varepsilon)\|f\|\|g\| \text { for any } f, g \text { in } A,
$$

where $f \circ g=\tilde{T}^{-1}(T f \cdot T g)$.

Notice that Theorem 1 does not settle the question whether small algebraic and metric perturbations of a function algebra produce the same class of algebras in a limit situation. More precisely, Theorem 1 does not show whether the Banach-Mazur distance between two function algebras is equal to one if and only if these algebras are algebraically isomorphic. The answer is negative and can be found in [2]. 


\section{METRIC ALGEBRAIC PERTURBATIONS OF FUNCTION ALGEBRAS}

\section{REFERENCES}

1. R. A. Hirschfeld and W. Zelazko, On spectral norm Banach algebras, Bull. Acad. Polon. Sci. 16 (1968), 195-199.

2. K. Jarosz, Perturbations of uniform algebras, Bull. London Math. Soc. 15 (1983) 133-138.

3. B. E. Johnson, Perturbations of Banach algebras, Proc. London Math. Soc. 34 (1977), 439-458.

4. R. V. Kadison and D. Kastler, Perturbations of von Neumann algebras I, Stability of type, Amer. J. Math. 94 (1972), 38-54.

5. M. Nagasawa, Isomorphisms between commutative Banach algebras with an application to rings of analytic functions, Kodai Math. Sem. Rep. 11 (1959), 182-188.

6. J. Phillips, Perturbations of $C^{*}$-algebras, Indiana Univ. Math. J. 23 (1973-74), 1167-76.

7. I. RAEBuRn and J. L. TAYloR, Hochschild cohomology and perturbations of Banach algebras, J. Func. Anal. 25 (1977), 258-266.

8. R. Rochierg, Deformations of uniform algebras, Proc. London Math. Soc. 39, (1979), 93-118.

InSTITUTE OF MATHEMATICS

WARSAW UNIVERSITY

Warsaw, Poland 